\title{
Las mujeres comunicadoras y relacionistas públicas en Costa Rica. Una aproximación a su perfil profesional.
}

Female communication and public relations practitioners in Costa Rica. An approximation to their professional profile.

\section{Praticantes de comunicação e relações públicas femininas na Costa Rica. Uma aproximação ao perfil profissional deles.}

\author{
Nadia Khalil Tolosa ${ }^{1}$ \\ Universidad Rey Juan Carlos (España) \\ nadia.khalil@urjc.es \\ Cristina Fuentes Lara ${ }^{2}$ \\ Universidad Rey Juan Carlos (España) \\ cristina.fuentes@urjc.es \\ Piet Verhoeven ${ }^{3}$ \\ Universidad de Ámsterdam (the Netherlands) \\ p.verhoeven@uva.nl
}

Fecha de recepción: 06 de febrero de 2018

Fecha de recepción evaluador: 30 de septiembre de 2018

Fecha de recepción corrección: 06 de noviembre de 2018

\footnotetext{
${ }^{1}$ Nadia Khalil Tolosa es Licenciada en: Traducción e Interpretación, Ciencias Políticas y de la Administración.

2 Cristina Fuentes Lara es Doctora en Estudios Migratorios. Licenciada en Ciencias Políticas y de la Administración y Licenciada en Sociología. https://orcid.org/0000-0003-3198-7701

${ }_{3}^{3}$ Piet Verhoeven es profesor adjunto de Comunicación Corporativa en la Escuela de Investigación de Comunicación de Amsterdam y en la Escuela de Posgrado de Ciencias de la Comunicación.
} 


\title{
Resumen
}

Este artículo analiza la situación de las mujeres profesionales del ámbito de la gestión de la comunicación y las relaciones públicas en Costa Rica. Centrándonos en aspectos de corte estructural, el objetivo es estudiar la feminización de la profesión en el país, así como la existencia del denominado techo de cristal y de diferencias salariales entre hombres y mujeres. Finalmente, también se analizan los conocimientos de los profesionales costarricenses en cuanto a las nuevas tecnologías relacionadas con el big data.

Se ha realizado un análisis estadístico con los datos cuantitativos de profesionales costarricense obtenidos en el proyecto Latin American Communication Monitor (20162017). Los resultados dan cuenta de la existencia mayoritaria de mujeres en la profesión, así como de la brecha salarial entre hombres y mujeres, del techo de cristal que limita el acceso de las mujeres a los puestos directivos y de un mayor conocimiento de las tendencias en comunicación por parte de las mujeres.

Palabras clave: relacionistas públicas, brecha salarial, techo de cristal, big data, perfil profesional

\begin{abstract}
This paper analyzes the female practitioners' situation in the field of the communication management and public relations in Costa Rica. Focused on structural aspects, the aim is to study the feminization of the industry in the country, as well as the existence of the socalled glass ceiling and the salary gap between men and women. Finally, we also analyze the Costa Rican practitioners' knowledge regarding new technologies related to big data.

A statistical analysis with the quantitative data about Costa Rican practitioners from the project Latin American Communication Monitor (2016-2017) has been used. Results show the women's majority existence in the field, the salary gap between men and women, the glass ceiling limiting women's access to managerial positions, and women's greater knowledge about trends in communication.
\end{abstract}

Keywords: female practitioners, salary gap, glass ceiling, big data, professional profile

\section{Resumo}

Este artigo analisa a situação das praticantes femininas no campo da gestão de comunicação e relações públicas na Costa Rica. Focado em aspectos estruturais, o objetivo é estudar a feminização da indústria no país, bem como a existência do chamado teto de vidro e a diferença salarial entre homens e mulheres. Por fim, analisamos também o conhecimento dos profissionais da Costa Rica em relação às novas tecnologias relacionadas ao Big Data.

Foi utilizada uma análise estatística com dados quantitativos sobre os praticantes costarriquenhos do projeto Latin American Communication Monitor (2016-2017). Os resultados mostram a existência da maioria das mulheres no campo, a diferença salarial 
entre homens e mulheres, o teto de vidro que limita o acesso das mulheres a cargos gerenciais e o maior conhecimento das mulheres sobre as tendências na comunicação.

Palavras-chave: praticantes do sexo feminino, lacuna salarial, teto de vidro, big data, perfil profissional

\section{Introducción}

Este artículo analiza algunos aspectos de la situación de las mujeres profesionales del ámbito de la gestión de la comunicación y las relaciones públicas en Costa Rica. Centrándonos en aspectos de corte estructural, estudiamos la feminización de la profesión en la región, así como la existencia del denominado techo de cristal y de diferencias salariales entre hombres y mujeres, además de analizar los conocimientos de los profesionales costarricenses en cuanto a las nuevas tecnologías relacionadas con el big data.

Costa Rica es uno de los 17 países que formó parte de la segunda edición del estudio Latin American Communication Monitor (LCM 2016/2017). Junto con el European Communication Monitor y el Asia-Pacific Communication Monitor constituye el mayor estudio sobre la profesión en el mundo basado en sólidos estándares empíricos. Este proyecto estudia en profundidad los diferentes aspectos de la profesión en la región de América Latina relacionados con las tendencias en comunicación estratégica y temas tales como big data, automatización, engagement, influencers, coaching y las competencias profesionales. Los datos nos permiten centrarnos en el caso particular costarricense, poco explorado en la literatura académica del campo que nos ocupa. Para ello, se realizará un análisis estadístico con los datos disponibles del estudio LCM 2016/2017.

El propósito de este trabajo es presentar una serie de hallazgos en torno a la profesión de la comunicación en Costa Rica, centrándonos, en la situación de las mujeres profesionales de esta área de acuerdo con unos determinados aspectos. Estos aspectos son, en primer lugar, la feminización de la profesión, que parece generalizada en todo el mundo; en segundo lugar, el tipo de cargo que ocupan las mujeres en este ámbito en Costa Rica, donde, de nuevo, existe una tendencia global según la cual las mujeres no suelen copar los puestos directivos más altos; en tercer lugar, se comprobará si siguiendo las tendencias globales las mujeres en Costa Rica tienen unos salarios inferiores a los de sus pares masculinos; y, en cuarto lugar, se indagará sobre el conocimiento que muestran las mujeres en torno a las vanguardias y tendencias tecnológicas, concretamente, sobre el big data, en comparación con los hombres.

Otros estudios previos ya indagan sobre estos aspectos de interés para la profesión, pero se centran en otras zonas geográficas. De acuerdo con Tench, Moreno, Khalil y Fuentes (2017), poco se sabe acerca de estas realidades en el subcontinente americano, donde no se han realizado estudios específicos sobre las discriminaciones de género en la profesión; al tiempo que también existe una carencia de datos empíricos y de literatura para apoyar la realidad observada de forma teórica. En esta línea, destacan los recientes trabajos sobre América Latina en su conjunto (Molleda, Moreno, Athaydes, 
\& Suárez, 2012; Moreno, Molleda, Athaydes \& Suárez, 2015; Moreno, Molleda, Athaydes, Suárez, Herrera \& Álvarez, 2017; Tench, Moreno, Khalil \& Fuentes, 2017; Tench, Topic \& Moreno, 2017). Por este motivo, un estudio sobre la situación de las mujeres profesionales de la comunicación en Costa Rica cobra especial relevancia.

\section{Marco referencial}

\section{Las mujeres en la gestión de la comunicación y las relaciones públicas}

Se considera que la diversidad de género es un factor clave para la excelencia en el ámbito de las relaciones públicas y la gestión de la comunicación (Tench et al., 2017c). Muchos autores se hacen eco del creciente número de profesionales mujeres en el campo de la gestión de la comunicación y las relaciones públicas en todo el mundo que, además, ya supera al número de hombres y se habla de una profesión feminizada (Andsager \& Hust, 2005; Wrigley, 2002; Aldoory \& Toth, 2002; Tench et al., 2017b). Los escasos estudios centrados en la región de América Latina también indican una mayor presencia de mujeres en la profesión. No obstante, continúan encontrándose diferencias de género en este ámbito laboral (véase, por ejemplo, Grunig, Toth \& Hon, 2000; Grunig, 2001; Place, 2011; Tench et al., 2017a; Tench et al., 2017b).

\section{Techo de cristal y diferencia salarial entre hombres y mujeres}

Otro aspecto de interés al analizar el perfil de las mujeres en el ámbito de las relaciones públicas y la gestión de la comunicación es la implicación que el mero hecho de ser mujer puede llegar a tener en la carrera profesional de estas y sus retribuciones económicas. El denominado techo de cristal considera que la condición de ser mujer limita el ascenso en la carrera profesional de las mujeres, impidiendo que ocupen los puestos de mayor rango en las organizaciones. El término fue acuñado originalmente en 1986 y es una metáfora para describir las barreras invisibles a las que las mujeres deben hacer frente cuando pretender ascender en sus puestos de trabajo (Hymowitz \& Schellhardt, 1986). Más concretamente, el techo de cristal se puede definir como el fenómeno en que las mujeres desaparecen a medida que se asciende en la organización jerárquica de una empresa (Fernández \& Campero, 2017, p. 74). Pese a que el término describía la realidad de hace unas décadas, continúa siendo muy conocido en cualquier ámbito y se siguen encontrando muestras de su existencia, también en el campo de las relaciones públicas (véase Aldoory \& Toth, 2002, p. 106). Las razones para su persistencia radican en diferentes problemas como la socialización de los roles de género o la selección y el desarrollo profesional de las mujeres en las organizaciones (Purcell \& Baldwin, 2003, p. 218).

Estudios recientes muestran que la flexibilidad horaria y el denominado teletrabajo pueden ser de gran utilidad para que las mujeres consigan mantener sus trabajos y continuar su carrera profesional después de ser madres (Chung \& van der Horst, 2017). Sin embargo, estas condiciones no son siempre posibles. Autoras como D. Pompper (2007) destacan la posición desventajosa de las mujeres por la existencia de desigualdades sociales, políticas y económicas por cuestión de género en las organizaciones. En esta línea, algunos autores consideran que los patrones del techo de 
cristal pueden ser también resultado de los procesos de selección y contratación externa y no sólo sesgos de la promoción interna de las organizaciones (Fernández \& Campero, 2017).

La existencia de una mayoría de mujeres en el campo de las relaciones públicas no logra evitar que estas obtengan un salario inferior al de sus compañeros hombres tal y como reflejan diversos estudios y auditorías profesionales (Tench et al., 2017b; Andsager \& Hust, 2005; Aldoory \& Toth, 2002; Wrigley, 2002; Choi \& Hon, 2002). Ello supone que, además de existir diferencias en cuanto a los tipos de puestos que ocupan hombres y mujeres, también se encuentran diferencias salariales. Igualmente ocurre en la región de América Latina (Tench et al., 2017a).

Diversos estudios dan cuenta de la significativa diferencia salarial que sigue existiendo en todo el mundo en este ámbito de la gestión de la comunicación y las relaciones públicas. Así, por ejemplo, estudios como el European Communication Monitor (Zerfass et al., 2007, 2008, 2009, 2010, 2011, 2012, 2013, 2014, 2015, 2016) confirman estas diferencias en la remuneración económica en todo tipo de cargos, desde los puestos directivos a los miembros de equipo en el contexto europeo. Si bien son pocas las mujeres que ocupan puestos directivos superando el techo de cristal, sus salarios suelen ser más bajos que los de sus pares masculinos; también, en América Latina (Tench et al., 2017a).

\section{Tendencias en la comunicación: conocimiento sobre big data}

El último aspecto del perfil profesional que se analiza en este artículo es el conocimiento de las vanguardias tecnológicas. En concreto, una de las grandes tendencias actuales en la comunicación estratégica es el big data. El uso de algoritmos ya está modificando el mundo de la gestión de la comunicación y las relaciones públicas, así como la comunicación en general, y se espera que en los próximos años su desarrollo e implementación aumenten considerablemente. De ahí surge la necesidad de investigar hasta qué punto los/as comunicadores/as tienen conocimientos respecto a si son conscientes de la importancia que el big data representa en su ámbito de trabajo. Esta información es de gran utilidad a la hora de definir su perfil profesional.

El debate sobre la introducción del big data en el ámbito de la comunicación estratégica y las relaciones públicas es muy reciente (Weiner \& Kochar, 2016). Pese a la gran importancia que puede llegar a tener para los profesionales, que deberían estar al tanto de lo que su uso puede implicar, es un tema muy poco tratado en la literatura académica (Zerfass et al., 2016). El término big data se refiere a las bases de datos que generan la actividad humana y las nuevas tecnologías con, por ejemplo, los dispositivos móviles y herramientas de seguimiento (Lewis, Zamith \& Hermida, 2013).

Además, son bases de datos que no se pueden tratar con las herramientas tradicionales por su enorme tamaño y complejidad (Guo, Vargo, Pan, Ding \& Inshwar, 2016). Así pues, su uso puede suponer un cambio cualitativo en la profesión, pero presenta también un gran número de desafíos (Sivarajah, Kamal, Irani \& Weerakkody, 2017). Se trata, por tanto, de un fenómeno transversal, ya que implica a diferentes 
departamentos, que facilita "conocer los avances y cambios tecnológicos que están alterando los modelos de producción, consumo y comportamiento de las sociedades" (Dircom, 2017, p. 98). Por todo ello, es relevante conocer el grado de uso y conocimiento que muestran los profesionales de la comunicación y las relaciones públicas al respecto. Asimismo, es interesante apuntar que el uso del big data puede incidir en las relaciones y la cohesión de todos los países de la región de América Latina (Moreno et al., 2017).

\section{La profesión de la gestión de la comunicación y las relaciones públicas en Costa Rica}

De acuerdo con Mayela Fallas (2014, p. 46-47), los países del área de América Central, como Costa Rica, están inmersos en los procesos de globalización actuales y a lo largo de su historia tanto la influencia española como, más recientemente, la estadounidense ha sido relevantes. Así, de acuerdo con esta autora (Mayela Fallas, 2014, p. 47), la actividad y el desarrollo de los relacionistas públicos en la región de América Central y las diferencias entre los países que la forman viene marcada en gran medida por la influencia de otras naciones a lo largo de su historia y la actualidad.

El campo de las relaciones públicas en Costa Rica ha experimentado un crecimiento sin interrupción desde sus inicios, que algunos autores ubican al término de la Guerra Civil de 1948, con la creación y la consolidación de instituciones autónomas en materia de salud, seguridad, finanzas y telecomunicaciones, entre otras, y, paralelamente, el desarrollo de las relaciones públicas con el establecimiento de oficinas para explicar estos servicios, generar información sobre asuntos de interés en los medios, trabajar en la comunicación interna, la imagen del liderazgo de la organización y también la organización de eventos formales (Mayela Fallas, 2014, p. 51-51).

Aunque inicialmente las prácticas de la profesión se centraban en las relaciones con los medios de comunicación, han ido ampliándose de manera progresiva para alcanzar un nivel más completo y estratégico de desarrollo que ya incluye todas las áreas de las relaciones públicas modernas (Mayella Fallas, 2014, p. 53).

\section{Metodología}

La metodología que se ha llevado a cabo en esta investigación ha sido de corte cuantitativo, ya que expresa la realidad social en términos matemáticos, así como las relaciones existentes entre ellos (López-Doblas, 2005). La técnica de producción de datos desarrollada fue un cuestionario online que se distribuyó entre las personas profesionales de comunicación y relaciones públicas en Costa Rica.

Este cuestionario es parte de una investigación más amplia, el proyecto Latin American Communication Monitor (LCM) 2016-2017. El cuestionario del LCM, además de en Costa Rica, se implementó en otros 16 países latinoamericanos: Argentina, Bolivia, Brasil, Chile, Colombia, Ecuador, El Salvador, Guatemala, Honduras, México, Panamá, Paraguay, Perú, República Dominicana, Uruguay y Venezuela. La muestra total de la investigación fue de 914 casos. Concretamente en Costa Rica, que es el país objeto de esta investigación, se extrajeron 50 casos. 
Llegados a este punto, los datos obtenidos mediante el cuestionario fueron codificados (Bardin, 1986) con el propósito de prepararlos para el análisis estadístico. La técnica de análisis de los datos que se empleó ha sido el análisis descriptivo al considerarse como la más adecuada para el tipo de muestra. La elección se debe a que el análisis descriptivo tiene como finalidad estudiar una información específica de un determinado grupo -en esta investigación, las personas profesionales de comunicación y relaciones públicas en Costa Rica- a partir de una serie de variables que son influyentes para describir el objeto de la investigación (Hernández et al., 2007).

Respecto a los análisis estadísticos realizados a las variables, se ha optado por combinar métodos - univariantes, bivariantes y multivariantes- de acuerdo con dos criterios: el tipo de variables y los objetivos que se pretendían alcanzar con el análisis estadístico. Desagregando los análisis, se han utilizado métodos univariantes distribución de frecuencias-, bivariantes -tabla de contingencia- y multivariantes análisis clúster-. Los análisis bivariantes y multivariantes se han testado estadísticamente con Chi cuadrado de Pearson $\left(\mathrm{X}^{2}\right)$. Para esta investigación se han considerado resultados significativos entre las variables cuando $\mathrm{p} \leq 0,05$, y altamente significativos en los casos en los que $\mathrm{p} \leq 0,01$.

Los datos del cuestionario se han analizado estadísticamente con el software informático Statistical Package for the Social Science (SPSS) en la versión 23 del software.

Los hallazgos de la investigación se estructuran en dos secciones. Para la primera sección referente al perfil de la comunicadora en Costa Rica, las variables empleadas para el análisis han sido: sexo, cargo dentro de la empresa, tipo de empresa en la que trabaja y salario anual. Para esta sección los análisis realizados fueron análisis de frecuencias y tablas de contingencia. En la segunda sección, las mujeres costarricenses en el big data, se realizado un análisis clúster basado en las variables sobre la atención prestada al debate sobre el big data, y el conocimiento sobre las definiciones del big data (Moreno et al., 2017, p. 30).

\section{Hallazgos de la investigación}

Los principales resultados de la investigación se estructuran alrededor de dos secciones. La primera de ellas tiene como finalidad dilucidar cuál es el perfil de la mujer profesional de la comunicación y las relaciones públicas en Costa Rica; mientras que la segunda sección es de corte más analítico e indaga en la postura sobre el big data que tiene la mujer costarricense.

\section{Sección 1: El perfil de la comunicadora en Costa Rica}

La distribución de frecuencias muestra cómo la profesión de la comunicación se encuentra feminizada, de tal forma que algo más de tres cuartas partes de la muestra son mujeres. 
Tabla 1: Distribución de frecuencias del sexo de las personas encuestadas

\begin{tabular}{lll}
\hline & Frecuencia & Porcentaje \\
\hline Hombres & 12 & $24 \%$ \\
Mujeres & 38 & $76 \%$ \\
Total & 50 & $100 \%$ \\
\hline
\end{tabular}

Fuente: Elaboración propia a partir de los datos del LCM (2016-2017).

Sin embargo, si indagamos sobre qué puestos de trabajo ocupan estas mujeres y en qué se diferencian de los hombres en el organigrama de la empresa o agencia de comunicación, se aprecia que las mujeres ocupan los puestos de más bajo rango. De hecho, el 40,5\% de las mujeres son miembros de equipo o consultoras, por un 33,3\% de los hombres en esta ocupación laboral. Si bien es cierto que más de un tercio de las mujeres ocupan posiciones de dirección, no parece tan significativo en comparación con el $25 \%$ de hombres que también ostentan este cargo. De tal forma, que los hombres son minoritarios en la profesión, pero ocupan puestos de responsabilidad como jefes de equipo o de unidad $(41,7 \%)$ o directores del departamento de comunicación o CEO de agencia (25\%); mientras que cuatro de cada diez las mujeres en la profesión en Costa Rica ostentan cargos de miembro de equipo o consultoras.

Tabla 2: Cargo en la empresa cruzado con el sexo

\begin{tabular}{|c|c|c|c|c|}
\hline & & Hombre & Mujer & Total \\
\hline $\begin{array}{lc}\text { Director del } & \text { departamento } \\
\text { comunicación } & \text { corporativa } \\
\text { organizacional/CEO de agencia }\end{array}$ & $\begin{array}{r}\mathrm{de} \\
\mathrm{u}\end{array}$ & $25 \%$ & $35,1 \%$ & $32,7 \%$ \\
\hline Jefe de equipo/Jefe de unidad & & $41,7 \%$ & $24,3 \%$ & $28,6 \%$ \\
\hline Miembro de equipo/Consultor & & $33,3 \%$ & $40,5 \%$ & $38,8 \%$ \\
\hline Total & & $100 \%$ & $100 \%$ & $100 \%$ \\
\hline
\end{tabular}

Fuente: Elaboración propia a partir de los datos del LCM (2016-2017). Significación estadística $p \leq 0,01$.

Y, por consiguiente, las mujeres perciben un salario menor que los hombres en este sector ocupacional. Casi un $85 \%$ de las mujeres cobran menos de 30.000 dólares anuales. 
Tabla 3: Salario anual cruzado con el sexo

\begin{tabular}{llll}
\hline & Hombre & Mujer & Total \\
\hline Menos de 30.000 dólares anuales & $75 \%$ & $84,2 \%$ & $82 \%$ \\
30.000 a 60.000 dólares anuales & $25 \%$ & $10,5 \%$ & $14 \%$ \\
Más de 200.000 dólares anuales & $0 \%$ & $2,6 \%$ & $2 \%$ \\
No contesta & $0 \%$ & $2,6 \%$ & $2 \%$ \\
Total & $100 \%$ & $100 \%$ & $100 \%$
\end{tabular}

Fuente: Elaboración propia a partir de los datos del LCM (2016-2017). Significación estadistica $p \leq 0,05$.

Ahondando en el perfil de la comunicadora en Costa Rica, se observa cómo prácticamente la mitad de las mujeres de la profesión $(48,6 \%)$ trabajan en empresas estatales, del sector público u organizaciones políticas. Precisamente, en este tipo de empresas es donde las mujeres logran ocupan puestos de responsabilidad, tanto es así que el 46,2\% de las mujeres directoras del departamento de comunicación trabajan para empresas estatales. Por el contrario, las empresas privadas son aquellas que menos cuentan con mujeres directivas.

Tabla 4: Tipo de empresa cruzado por el cargo en la empresa en el caso de las mujeres

\begin{tabular}{|c|c|c|c|c|}
\hline & $\begin{array}{c}\text { Director del } \\
\text { departamento de } \\
\text { comunicación } \\
\text { corporativa } \mathrm{u} \\
\text { organizacional/CEO } \\
\text { de agencia }\end{array}$ & $\begin{array}{c}\text { Jefe de } \\
\text { equipo/ } \\
\text { Jefe de unidad }\end{array}$ & $\begin{array}{l}\text { Miembro de } \\
\text { equipo/ } \\
\text { Consultor }\end{array}$ & Total \\
\hline Empresa privada & $7,7 \%$ & $22,2 \%$ & $13,3 \%$ & $13,5 \%$ \\
\hline $\begin{array}{l}\text { Empresa estatal, sector público } \\
\text { u organización política }\end{array}$ & $46,2 \%$ & $44,4 \%$ & $53,3 \%$ & $48,6 \%$ \\
\hline $\begin{array}{l}\text { Organización sin ánimo de } \\
\text { lucro o asociación }\end{array}$ & $23,1 \%$ & $11,1 \%$ & $13,3 \%$ & $16,2 \%$ \\
\hline $\begin{array}{l}\text { Agencia, asesoría o consultora } \\
\text { de comunicación o relaciones } \\
\text { públicas }\end{array}$ & $15,4 \%$ & $22,2 \%$ & $0 \%$ & $10,8 \%$ \\
\hline $\begin{array}{l}\text { Consultor freelance o } \\
\text { independiente }\end{array}$ & $7,7 \%$ & $0 \%$ & $20 \%$ & $10,8 \%$ \\
\hline Total & $100 \%$ & $100 \%$ & $100 \%$ & $100 \%$ \\
\hline
\end{tabular}

Fuente: Elaboración propia a partir de los datos del LCM (2016-2017). Significación estadistica $p \leq 0,05$.

\section{Sección 2: Las mujeres costarricenses y el big data}

El clúster mide en función de las respuestas a dos variables -sobre la atención prestada al debate sobre el big data, y el conocimiento sobre las definiciones del big data (Moreno et al., 2017)-, con el propósito de evaluar la comprensión completa del big data de las personas profesionales de la comunicación, incluyendo sus características de 
volumen, variedad, velocidad y veracidad (Chen, Chiang \& Storey, 2012; Gandomi \& Haider, 2015; Moreno et al., 2017; Schroeck, Shockley, Smart, Romero-Morales \& Tufano, 2012). En función de las respuestas de los y las comunicadoras se ha realizado un análisis clúster para clasificar a las personas encuestadas en cuatro grupos: informados, aspirantes, transeúntes y desinformados.

\section{Figura 1: Representación de clúster por conglomerados}

\section{MUCHO CONOCIMIENTO SOBRE BIG DATA}

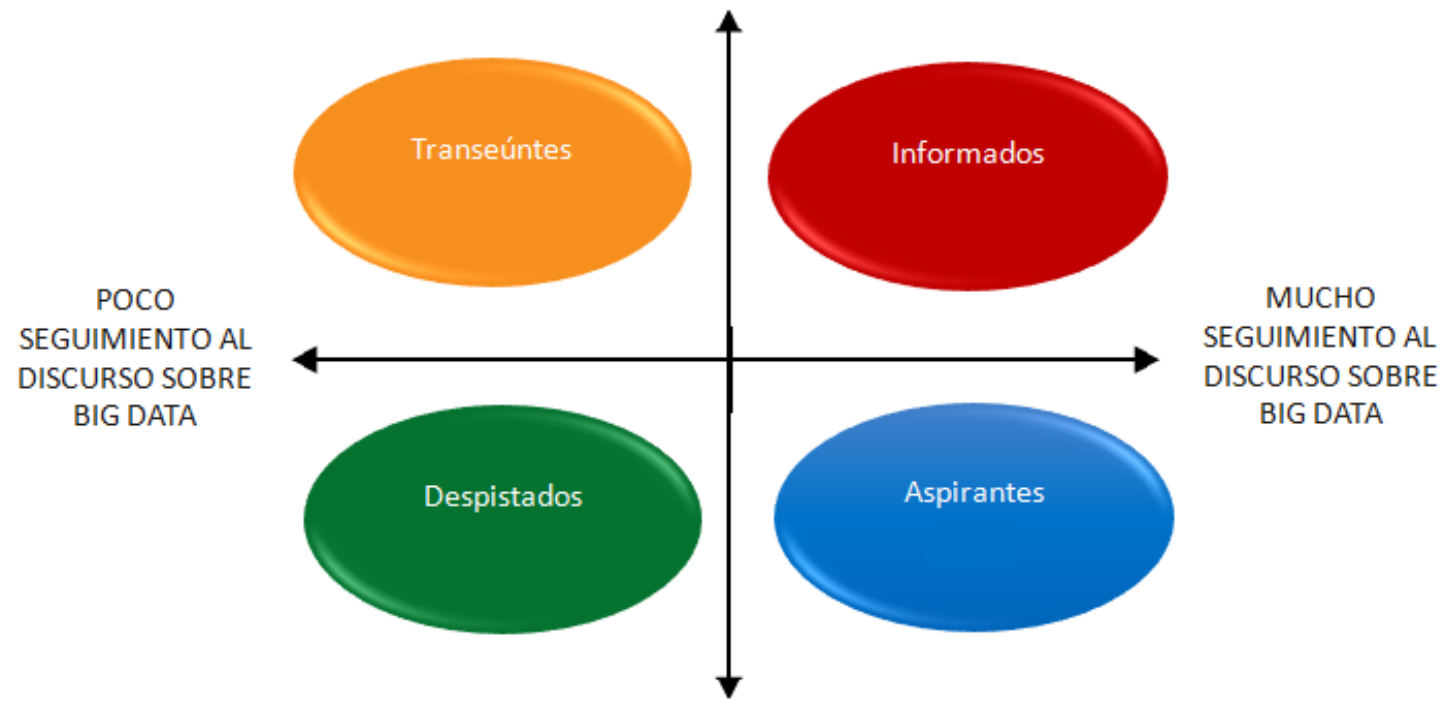

POCO CONOCIMIENTO SOBRE BIG DATA

Fuente: Elaboración propia a partir de los datos del LCM (2016-2017).

El análisis por sexo muestra cómo las mujeres costarricenses ocupan mayoritariamente $(35,1 \%)$ el conglomerado de las personas informadas -mucho conocimiento sobre big data y mucho seguimiento sobre el debate de big data-, por encima de los hombres costarricenses (20\%). Por el contrario, el conglomerado de los profesionales de la comunicación que son transeúntes -mucho conocimiento sobre big data y poco seguimiento sobre el debate de big data-, es en el que las mujeres comunicadoras son minoritarias $(10,8 \%)$ a diferencia de los hombres profesionales de la comunicación, donde una quinta parte de ellos se sitúan en ese estrato.

Tabla 5: Clúster de big data por sexo

\begin{tabular}{lccc}
\hline & Hombre & Mujer & Total \\
\hline Transeúntes & $20 \%$ & $10,8 \%$ & $12,8 \%$ \\
Aspirantes & $40 \%$ & $32,4 \%$ & $34 \%$ \\
Informados & $20 \%$ & $35,1 \%$ & $31,9 \%$ \\
Despistados & $20 \%$ & $21,6 \%$ & $21,3 \%$ \\
Total & $100 \%$ & $100 \%$ & $100 \%$ \\
\hline
\end{tabular}

Fuente: Elaboración propia a partir de los datos del LCM (2016-2017). Significación estadística $p \leq 0,05$. 


\section{Conclusiones}

La revisión bibliográfica y el análisis cuantitativo de los datos evidencian la diferenciación entre hombres y mujeres profesionales de la comunicación y relaciones públicas en Costa Rica. Si atendemos al perfil sociolaboral de las mujeres costarricenses del sector, se aprecia que, pese a ser una profesión principalmente dominada por mujeres, ni salarialmente ni en puestos de dirección se ven estas representadas cuantitativamente en comparación con los hombres.

Profundizando en los tipos de empresas donde principalmente desarrollan su actividad laboral las mujeres profesionales de la comunicación y relaciones públicas, se observa cómo son las empresas públicas quienes contratan a un mayor volumen de mujeres del sector. De hecho, es precisamente en este tipo de empresas donde las mujeres alcanzan, en mayor medida que en el resto de las organizaciones empresariales, los puestos de dirección del departamento de comunicación corporativa u organizacional. La explicación a tal situación es debida a que el acceso a las empresas públicas en Costa Rica se realiza por concurso público u oposición pública, lo cual reduce los posibles efectos del sesgo de género en la contratación.

Un factor que respalda la existencia de una diferenciación de género en la contratación, promoción y salario en el sector de la comunicación y de las relaciones públicas es el hecho de que las mujeres costarricenses ocupen principalmente el conglomerado de las personas informadas y capacitadas sobre big data. Sin embargo, este dato unido a la feminización de la profesión no se traduce en que las mujeres lleguen, al menos no en la misma proporción, a alcanzar puestos de dirección y mejores remuneraciones económicas.

Si bien las investigaciones que versan sobre el techo de cristal y los propios datos de esta investigación marcan una tendencia que apunta a que la discriminación por género es uno de los principales factores que limita a las mujeres costarricenses a ocupar posiciones de mayor responsabilidad y prestigio social en la profesión, hay que tener en consideración las limitaciones propias de esta investigación.

Por tanto, para futuras investigaciones sobre el big data y el papel de la mujer costarricense profesional de la comunicación y las relaciones públicas, se considera pertinente ampliar el número de casos de la investigación y la diversidad de perfiles, ya que ambas han sido limitaciones que se han detectado en el transcurso del análisis de los datos de esta investigación. 


\section{Bibliografía}

Aldoory, L. \& Toth, E. L. (2002), "Gender Discrepancies in a Gendered Profession: A Developing Theory for Public Relations", Journal of Public Relations Research, Vol. 14, No. 2, pp. 103-126.

Andsager, J. L. \& Hust, S. J. T. (2005) "Differential gender orientation in public relations: Implications for career choices", Public Relations Review, Vol. 31, pp. 85-91

Bardin, L. (1986). El análisis de contenido. Madrid: Akal.

Chen, H., Chiang, R. H. L. \& Storey, V. C. (2012). Business intelligence and analytics: from big data to big impact. MIS Quarterly, 36(4), 1165-1188.

Choi, Y. \& Hon, L. C. (2002), "The Influence of Gender Composition in Powerful Positions on Public Relations Practitioners' Gender-Related Perceptions", Journal of Public Relations Research, Vol. 14, No. 3, pp. 229-263.

Chung, H. \& van der Horst, M. (2017). Women's employment patterns after childbirth and the perceived access to and use of flexitime and teleworking. Human relations 0 (00), pp. 1-26. DOI: 10.1177/0018726717713828

Dircom (2017). Anuario de la Comunicación 2017. Madrid, España: Dircom.

Fernandez, R.M. \& Campero, S., (2017), "Gender sorting and the glass ceiling in hightech firms", ILR Review, Vol. 70, No. 1, pp.73-104.

Gandomi, A. \& Haider, M. (2015). Beyond the hype: Big data concepts, methods, and analytics. International Journal of Information Management, 35(2), 137-144.

Grunig, J. (2001), "The role of public relations in management and its contribution to organizational and social effectiveness", speech delivered in Taipei, Taiwan, May 12, 2001. Available at: http://www.instituteforpr.org/wpcontent/uploads/2001_PRManagement.pdf (accessed 12 December 2017).

Grunig, L. A., Toth, E. L. \& Hon, L. C. (2000), "Feminist Values in Public Relations", Journal of Public Relations Research, Volume 12, Issue 1, pp. 49-68

Guo, L., Vargo, C. J., Pan, Z., Ding, W. \& Ishwar, P. (2016). Big Social Data Analytics in Journalism and Mass Communication: Comparing Dictionary-Based Text Analysis and Unsupervised Topic Modleing. Journalism \& Mass Communication Quarterly, 1-28. doi: 10.1177/1077699016639231

Hernández R., Fernández, F. \& Baptista, P. (2007). Metodología de la investigación. México: McGraw Hill.

Hymowitz, C., \& Schellhardt, T. D. (1986) “The glass ceiling”, Wall Street Journal, 24 March, pp. 1D, 4D. 
Lewis, S. C., Zamith, R., \& Hermida, A. (2013). Content Analysis in an Era of Big Data: A Hybrid Approach to Computational and Manual Methods. Journal of Broadcasting \& Electronic Media, 57(1), 34-52. doi:10.1080/08838151.2012.76170

López Doblas, J. (2005). Herramientas para el trabajo sociológico. En Iglesias de Ussel, J. \& Trinidad, A. (eds.), Leer la sociedad. Una introducción a la sociología general. Madrid: Tecnos

Mayela Fallas, C. (2014). "Central America”. En Watson, T. (ed.) (2014). Latin American and Caribbean Perspectives on the Development of Public Relations: Other Voices, Palgrave Macmillan: Londres

Molleda, J. C., Moreno, Á., Athaydes, A., \& Suárez, A. M. (2012), "Macroencuesta latinoamericana de comunicación y relaciones públicas", Revista Organicom, Vol. 7, No. 13, pp. 119-141.

Moreno, A., Molleda, J. C., Athaydes, A., Suárez, A. M., Herrera, M. \& Álvarez, A. (2017). Latin American Communication Monitor 2016-2017. Tendencias en comunicación estratégica: big data, automatización, engagement, influencers, coaching y competencias. Resultados de una encuesta en 17 países. Madrid, España: EUPRERA/DIRCOM

Moreno, A., Molleda, J.C., Athaydes, A. \& Suárez, A.M. (2015), Latin American Communication Monitor 2015. Excelencia en comunicación estratégica, trabajo en la era digital, social media y profesionalización. Resultados de una encuesta en 18 países. EUPRERA, Brussels, Belgium.

Place, K. R. (2011), "Power and gender at the crossroads: A qualitative examination of the nexus of power and gender in public relations", PRism, Vol. 8, No. 1, pp. 113.

Pompper, D. (2007), "The Gender-Ethnicity Construct in Public Relations Organizations: Using Feminist Standpoint Theory to Discover Latinas' Realities”, Howard Journal of Communications, Vol. 18, No. 4, pp. 291-311.

Purcell, M. \& Baldwin, J.N. (2003), "The Relationship Between Dependent Care Responsibility and Employee Promotions", Review of Public Personnel Administration, Vol. 23, No. 3, pp. 217-240.

Schroeck, M., Shockley, R., Smart, J., Romero-Morales, D. \& Tufano, P. (2012). Analytics: The real-world use of big data. How innovative enterprises extract value

uncertain

data.

Recuperado

from

https://www.ibm.com/smarterplanet/global/fles/se__sv

se_intelligence_Analytics_-_The_real-world_use_of_big_data.pdf 
Sivarajah, U., Kamal, M. M., Irani, Z., \& Weerakkody, V. (2017). Critical analysis of Big Data challenges and analytical methods. Journal of Business Research, 70, 263286.

Tench, R., Moreno, A., Khalil, N. \& Fuentes, C. (2017a). "From intra-organizational to extra-organizational gender gaps. Creative methodological instruments to understand gender issues in Public Relations and Communication Management in Latin America”. 19th Annual EUPRERA Congress, Public Relations and the Power of Creativity Strategic opportunities, innovation and critical challenges, 12th-14th October, London.

Tench, R., Topic, M. \& Moreno, A. (2017b). "Male and female communication, leadership styles and the position of women in public relations", Interactions: Studies in Communication \& Culture, Volume 8 Numbers 2 \& 3. doi: 10.1386/iscc.8.2-3.231_1

Tench, R., Verčič, D., Zerfass, A., Moreno, A. \& Verhoeven, P. (2017c), Communication Excellence - How to Develop, Manage and Lead Exceptional Communications, Palgrave Macmillan, London, United Kingdom.

Weiner, M. \& Kochhar, S. (2016). Irreversible: The public relations big data revolution [IPR Whitepaper]. Gainesville, FL: Institute for Public Relations.

Wrigley, B. J. (2002), “Glass Ceiling? What Glass Ceiling? A Qualitative Study of How Women View the Glass Ceiling in Public Relations and Communications Management", Journal of Public Relations Research, Vol. 14, No.1, pp. 27-55.

Zerfass, A.; Moreno, A.; Tench, R.; Verčič, D., \& Verhoeven, P. (2013). European Communication Monitor 2013. A Changing Landscape - Managing Crises, Digital Communication and CEO Positioning in Europe. Results of a Survey in 43 Countries. Brussels: EACD/EUPRERA, Helios Media.

Zerfass, A., Moreno, A., Tench, R., Verčič, D., \& Verhoeven, P. (2009). European Communication Monitor 2009. Trends in Communication Management and Public Relations. Results of a Survey in 34 Countries. Brussels: EACD/EUPRERA, Helios Media.

Zerfass, A., Moreno, A., Tench, R., Verčič, D., \& Verhoeven, P. (2008). European Communication Monitor 2008. Trends in Communication Management and Public Relations - Results and Implications. Leipzig: University of Leipzig/Euprera.

Zerfass, A., Tench, R., Verčič, D., Verhoeven, P., \& Moreno, A. (2014). European Communication Monitor 2014. Excellence in Strategic Communication - Key Issues, Ledership, Gender and Mobile Media. Results of a Survey in 42 Countries. Brussels: EACD/EUPRERA, Helios Media.

Zerfass, A., Tench, R., Verhoeven, P., Verčič, D., \& Moreno, A. (2010). European Communication Monitor 2010. Status Quo and Challenges for Public Relations 
in Europe. Results of an Empirical Survey in 46 Countries. Brussels: EACD/EUPRERA, Helios Media.

Zerfass, A., Van Ruler, B., Rogojinaru, A., Verčič, D., \& Hamrefors, S. (2007). European Communication Monitor 2007. Trends in Communication Management and Public Relations - Results and Implications. Leipzig: University of Leipzig/Euprera.

Zerfass, A., Verčič, D., Verhoeven, P., Moreno, A., \& Tench, R. (2015). European Communication Monitor 2015. Creating communication value through listening, messaging and measurement. Results of a Survey in 41 Countries. Brussels: EACD/EUPRERA, Helios Media.

Zerfass, A., Verčič, D., Verhoeven, P., Moreno, A., \& Tench, R. (2012). European Communication Monitor 2012. Challenges and Competencies for Strategic Communication. Results of an Empirical Survey in 42 Countries. Brussels: EACD/EUPRERA, Helios Media.

Zerfass, A., Verhoeven, P., Tench, R., Moreno, A., \& Verčič, D. (2011). European Communication Monitor 2011. Empirical Insights into Strategic Communication in Europe. Results of a Survey in 43 Countries. Brussels: EACD/EUPRERA, Helios Media.

Zerfass, A., Verhoeven, P., Moreno, A., Tench, R., \& Verčič, D. (2016), European Communication Monitor 2016. Exploring trends in big data, stakeholder engagement and strategic communication. Results of a survey in 43 Countries. Brussels: EACD/EUPRERA, Quadriga Media Berlin.

\section{Notas}

1 Nadia Khalil Tolosa es Licenciada en Traducción e Interpretación (2005) por la Universitat Jaume I y licenciada en Ciencias Políticas y de la Administración (2010) por la Universidad de Valencia. Máster en Democracia y Gobierno (2011) por la Universidad Autónoma de Madrid y doctoranda en Ciencia Política en esta misma universidad. Profesora colaboradora en la Universidad Internacional de Valencia y miembro del Grupo de Estudios Avanzados en Comunicación (GEAC) de la Universidad Rey Juan Carlos. Colaboradora honorífica de la Universidad Rey Juan Carlos.

2 M. Cristina Fuentes Lara es Doctora en Estudios Migratorios (2017) por la Universidad de Granada. Licenciada en Ciencias Políticas y de la Administración (2010) y Licenciada en Sociología (2012) por la Universidad de Granada. Profesora Asociada de la Universidad Rey Juan Carlos, y miembro del Grupo de Estudios Avanzados en Comunicación (GEAC) de la Universidad Rey Juan Carlos, y del Observatorio de la Realidad Sociofronteriza del Mediterráneo (OARS del Med) de la Universidad de Granada. Colaboradora honorífica de la Universidad Rey Juan Carlos.

3 Piet Verhoeven es profesor asociado de comunicación corporativa en la Escuela de Investigación de Comunicación de Ámsterdam (ASCoR) y en la Escuela de Posgrado de Ciencias de la Comunicación. Su investigación incluye estudios en comunicación corporativa y relaciones públicas, especialmente en relación con corporaciones y medios de comunicación, la dinámica de las noticias de negocios y la comunicación de crisis. Desde 2008, es miembro del equipo de investigación del European Communication Monitor (ECM), una encuesta anual entre los profesionales de la comunicación corporativa en Europa sobre tendencias y problemas en la profesión. Comenzó a trabajar como científico de la comunicación siguiendo una carrera en la práctica de relaciones públicas, entre otros, en el Aeropuerto Schiphol de Amsterdam. 